\title{
Fatal Pulmonary Mycobacterium Celatum Infection in an Immunocompetent Patient. An Eight-Year Follow-Up of the First Case, a Review of the Literature and a Report of Beta-2 Microglobulin as a Potential Indicator of Disease Severity
}

\section{S. Rustscheff, J. Darelid}

Department of Internal Medicine, Värnamo General hospital and Department of Infectious diseases, Ryhov County hospital, Jönköping, Sweden.

Email: stefan.rustscheff@lj.se

Received October $4^{\text {th }}$, 2013; revised November $3^{\text {rd }}, 2013$; accepted November $10^{\text {th }}, 2013$

Copyright (C 2013 S. Rustscheff, J. Darelid. This is an open access article distributed under the Creative Commons Attribution License, which permits unrestricted use, distribution, and reproduction in any medium, provided the original work is properly cited.

\begin{abstract}
Setting: Swedish county hospital. Objective: Pulmonary M. celatum infection has been previously described only twice in an immunocompetent individual with no pre-existing chest disease. We describe the first Swedish case report of unusual drug-related complications and review the literature. Discussion: Atypical mycobacteriosis is often difficult to treat. We describe a fatal case of $\mathrm{m}$ celatum pneumonia in a previously healthy patient, unusual side effects encountered during her treatment and also the report of the utility of beta-2-microglobulin as a way to measure disease activity in mycobacterial infections. Conclusions: M. celatum may cause fatal pneumonia in previously healthy individuals. Treating the disease may be fraught with difficulty and there is a risk for very severe side effects, all of which must be weighed before taking the decision to treat this disease.
\end{abstract}

Keywords: Mycobacterium Celatum; Immunocompenent

\section{Introduction}

Mycobacterium celatum was discovered in 1993 [1]. It belongs to the Runyon group of nonchromogens and has biochemical characteristics similar to $\mathrm{M}$. xenopii and $\mathrm{M}$. avium intracellulare. Mycolic acid liquid chromatogramphy (HPLC) or 16S rRNA sequencing was often performed for proper identification. However, modern assays utilizing reverse hybridization is available nowadays. It is a pathogen of various animals such as the domestic ferret [2] and the white-tailed trogon [3] and may also be found in clinically healthy aquarium fish [4].

The first human cases described by Tortoli and Piersimoni [5] were found among AIDS victims and a grand total of 28 patients have been reported since 1994. Infec-

\footnotetext{
*This paper has received support in the form of an unrestricted grant of four weeks of paid research leave to dr Stefan Rustscheff, a part of which has been used to write this paper. This paper is deemed not to need ethical approval from the ethics committee of Linköping University hospital, as it is a retrospective review of a clinical case.
}

tions in immunocompetent patients seem to be exceedingly rare. The first report of a localized lymphadenitis in a previously healthy boy, came in 1994 [6] and a second in a 22-month-old infant was in 2004 [7]. The first case of pulmonary infection in an immunocompetent individual was detected post mortem in a patient treated for suspected tuberculosis [8]. The second case was described from Maastricht in 1999, where a 61-year-old male was presented with a history of three years of weight loss, cough, malaise and had a cavity in the right upper lobe. This case was also misdiagnosed as M. tuberculosis due to a false positive AMTD test [9]. The next case described in Italy was a 63-year-old HIV negative lady who, however, had suffered from pulmonary tuberculosis at the age of 16 and, in addition, had developed bronchiectases [10]. We would like to describe the first fatal case of m celatum pneumonia in an immunocompetent individual with no predisposing chest pathology. 

of Beta-2 Microglobulin as a Potential Indicator of Disease Severity

\section{Case Report}

A 77-year-old unmarried former wet-nurse and ship's stewardess was in 2001 referred from her private practitioner to a chest clinic in southern Sweden. She was a native of Sweden but had lived in the United States for decades as well. She was a lifelong non-smoker.

She presented with a non-productive cough and $10 \mathrm{~kg}$ weight loss. Her chest X-ray showed extensive interstitial infiltrates in the right lung, discrete infiltrates in the left, and a cavitation of the right apex lung. A ventricular lavage was threefold negative on PCR for $M$. tuberculosis as was culture on Löwenstein-Jensens medium and the BACTEC 460 Radiometric system. Four sputum samples were negative on direct microscopy and PCR against $M$. tuberculosis. Her PPD was nevertheless $15 \mathrm{~mm}$. A bronchoscopy, a ventricular lavage and a sputum sample in 2002 yielded a NTM species both on Bactec and Löwenstein-Jensens medium which was identified as Mycobacterium avium-intracellulare by the Accuprobe kit. The strain unfortunately died before sensitivity tests could be performed. Nevertheless, ex juvantibus treatment was instituted.

Her initial ESR (sedimentation) was 77. She was put on Rifampin $450 \mathrm{mg}$, Isoniazid $300 \mathrm{mg}$ and ethambutol $800 \mathrm{mg}$ and followed. Her cough disappeared but due to rapidly progressive loss of vision, ethambutol was discontinued after 11 months of treatment. A central retinal vein thrombosis was found to be the cause of the loss of vision. A new bronchoscopy 2003 yielded a mycobacterium sensitive to clarithromycin, amikacin and ethambutol but resistant to ciprofloxacin and rifampicin. This turned out to be the first Swedish isolate of M. celatum. It was classified at Karolinska University using 16s rRNA sequencing.

A re-bronchoscopy 2004 yielded the same result, now using gas chromatography at the mycobacterial research laboratory, Lund University (Kristina Persson, BmA, pers.comm). The growth of $M$. celatum was considered to be a commensal. The patient had been losing weight constantly since 2001, going from $61 \mathrm{~kg}$ to $44 \mathrm{~kg}$. This was attributed to chewing difficulties due to a concomitant trigeminal neuralgia. Her chest $\mathrm{x}$-ray never showed resolution of the infiltrates and her ESR rose to 81. No treatment was instituted.

The patient moved to our area in 2005. She was referred to us from the first hospital for follow-up. She had an ESR of 90 and a dry cough. Her chest X-ray in May 2005 showed the same picture as in 2004 with right-sided perihilar fibrotic infiltrates and volume loss. A new bronchos copy was performed and yielded M. celatum, sensitive to amikacin, ofloxacin, clarithromycin and ethambutol. The strain was classified through reverse hybridi- zation by the commercial kit "Genotype CM/AS" (Hain Diagnostics) at Karolinska University. It was also successfully cultured on Löwenstein-Jensens medium and Bactec. Treatment was now instituted with amikacin 500 mg twice weekly, ethambutol and clarithromycin, $1 \mathrm{~g}$ per diem and moxifloxacin $400 \mathrm{mg}$ for two months, with the intention to reduce ethambutol to $600 \mathrm{mg}$ and discontinue amikacin thereafter, for a total treatment regime of at least 18 months.

We followed the patient and noted a reduction in ESR from 90 to 44 and a weight gain to $50 \mathrm{~kg}$. Unfortunately in January 2006 the patient suffered another retinal vein thrombosis in her healthy eye and was now clinically amaurotic. Ethambutol was discontinued in november 2006 because the patient still had some peripheral vision left and we did not want to risk it if ethambutol happened to be the cause of her retinal thromboses. Unfortunately, this decision soon led to a relapse in her pulmonary disease in april 2007.

She rose in ESR to 89 and developed new patchy infiltrates on her chest X-ray. A decision was taken to supplement her treatment with amikacin $500 \mathrm{mg}$ twice weekly again. 3 months afterwards we saw a resolution of the infiltrates and a return to previous levels in ESR, 42. She remained culture negative. Her cough disappeared and, in spite of her amaurosis she felt much better than before institution of treatment for her mycobacterial disease. We could not rule out that her recurrent retinal thromboses might be a side effect from ethambutol and she was kept off this drug. In spring 2008 she unfortunately suffered a relapse, making it necessary for us to reinstate treatment with ethambutol, moxifloxacin and amikacin in the same dosage as previously tolerated. This time, however, the patient developed severe hearing loss after having had carbamazepine prescribed by another source for trigeminal neuralgia. Unfortunately, the hearing loss was only partially reversible and the patient was now dependent on a hearing aid, as well as being amaurotic! All antimycobacterial drugs were discontinued in february 2009. Her ESR varied between 50 and 94 during 2009, while her chest X-ray showed a progressively floccular subhilar infiltrate with an atelectasis until September, when she was admitted with respiratory insufficiency and an $\mathrm{SaO}_{2}$ of $83 \%$. This X-ray actually showed partial resolution of the infiltrates but also oedema and cardiomegaly. She had a $40 \mathrm{~mm} \mathrm{Hg}$ right ventricular pressure on her echocardiography, a creatinine level of $113 \mathrm{mmol} / \mathrm{l}$, normal ultrasound examinations of her legs, a D dimer of 2.69 (limit 0.25) and a ventilation perfusion scintigraphy showing at least an intermediate likelihood of pulmonary embolism. Treatment with tinzaparin $20,000 \mathrm{E} / \mathrm{ml}, 0.7 \mathrm{ml} \cdot \mathrm{s} \cdot \mathrm{c}$ daily was instituted for 6 months. The patient could yet again be discharged without sup- 

of Beta-2 Microglobulin as a Potential Indicator of Disease Severity

plemental oxygen.

In august 2010 the patient returned to us, now again with a respiratory insufficiency and a fast atrial fibrillation. She was reinstituted on tinzaparin and also received loracarbef and furosemide. Sputum samples grew proteus mirabilis and treatment was changed to ciprofloxacin. Her ESR was only 32 and her CRP 36. Her x-ray showed progressive infiltrates in her right lower lobe. Nevertheless she regained her respiratory sufficiency and was discharged on September $13^{\text {th }}$ with an alleviated cough in order to wait for the results of the mycobacterial samples.

She was found dead in her sleep at her home on September $14^{\text {th }}$, 2010. Her sister refused an autopsy.

During the whole eight-year period we followed her beta-2 microglobulin serum levels. She had a reasonable kidney function for her age, with a creatinine clearance of 40 - 45 and no other known reasons for an elevated level of beta-2 microglobulin. Her levels before institution of treatment were $4.1 \mathrm{mg} / \mathrm{l}$ (ref 0.6 - 2.4). Treatment resulted in a rapid drop to $2.7 \mathrm{mg} / \mathrm{l}$. In april 2007, concomitant with her exacerbation, levels rose to $3.8 \mathrm{mg} / \mathrm{l}$. They then went down with treatment and hovered around $2.7 \mathrm{mg} / \mathrm{l}$ until autumn 2008, when she had another exacerbation and a concomitant rise to $5.4 \mathrm{mg} / \mathrm{l}$. On treatment levels declined to $2.9 \mathrm{mg} / \mathrm{l}$ and have on discontinuation of all therapy risen again to $3.9 \mathrm{mg} / \mathrm{l}$ in summer, 2009 . Unfortunately, no beta-2-microglobulin samples were measured on her last admission to our ward.

\section{Discussion}

Atypical mycobacteria may be found as commensals in the bronchial tree, but a surprising number of them are pathogens in a true sense. Treatment may be prolonged and very difficult.

A decision to treat non-tuberculous mycobacteria rests on repeated cultures of the same Specimen, or culture positive cases with progressive disease and no other known cause.

We know that $\mathrm{M}$ celatum may be misclassified as $\mathrm{M}$ tuberculosis with the Amplified M tuberculosis direct test [11]. We have been able to grow M. celatum on three occasions and the patient has responded to treatment, relapsed twice after amikacin was withdrawn, improved on its reinstitution, and, finally, succumbing to the disease when treatment was withheld. We cannot rule out that this patient first suffered from a mycobacterium avium-intracellulare infection but we deem it highly unlikely that this pathogen would be so easily treated that it would disappear after just six months of chemotherapy, never to return, especially when this patient immediately afterwards got colonized by M. celatum.

\section{Conclusions}

We deem that it is the first Swedish case of a Mycobacterium celatum infection, the first fatal case in a previously healthy individual, and the third case in the world of pulmonary infection in an immunocompetent patient with no previous lung disease, confirming that $M$. celatum is a true pathogen in man. The PPD test was strongly positive. Cultures, direct smears and PCR assays have been negative for $M$. tuberculosis. We therefore suggest that the PPD may cross-react with M. celatum. A negative Quantiferon Gold test has been performed in 2009. We postulate that central retinal vein thrombosis might be a rare side effect of ethambutol.

Unfortunately, the ototoxic effect of aminoglycosides is well known and may occur more frequently in the elderly, when treatment is prolonged and when other ototoxic drugs are added. We therefore advise the utmost caution when adding potentially ototoxic and/or nephrogenic drugs to an aminoglycoside-containing regimen, even if the aminoglycoside is given in a low total weekly dose. We would also like to point out the dangers of withholding treatment altogether in these infections.

Finally, we would like to draw attention to beta-2 microglobulin, which is said to be synthetised in granulomata. There is an excellent paper published on beta- 2 microglobulin levels in tuberculosis patients [12]. We tentatively postulate that this test could be of potential value in monitoring the course of non-tuberculous mycobacterial diseases.

\section{REFERENCES}

[1] W. R. Butler, S. P. O’Connor, M. A. Yakrus, R. W. Smithwick, B. B. Plikaytis, C. W. Moss et al., "Mycobacterium Celatum sp.nov," International Journal of Systematic Bacteriology, Vol. 43, No. 3, 1993, pp. 539-548. http://dx.doi.org/10.1099/00207713-43-3-539

[2] M. Valheim, B. Djönne, R. Heiene and D. A. Caugant, "Disseminated Mycobacterium Celatum (Type 3) Infection in a Domestic Ferret Mustela Putorius Furo)," Veterinary Pathology, Vol. 38, No. 4, 2001, pp. 460-463. http://dx.doi.org/10.1354/vp.38-4-460

[3] M. F. Bertelsen, C. Gröndahl and S. B. Giese, "Disseminated Mycobacterium Celatum Infection in a White-Tailed Trogon (Trogon Viridis),” Avian Pathology, Vol. 35, No. 4, 2006, pp. 316-319. http://dx.doi.org/10.1080/03079450600821133

[4] V. Beran, L. Matlova, L. Dvorska, P. Svastova and I. Pavlik, "Distribution of Mycobacteria in Clinically Healthy Ornamental Fish and Their Aquarian Environment," Journal of Fish Diseases, Vol. 29, No. 7, 2006, pp. 383393. http://dx.doi.org/10.1111/j.1365-2761.2006.00729.x

[5] C. Piersimoni, E. Tortoli, F. de Lalla, D. Nista, D. Donato, 
S. Bornigia, et al., "Isolation of Mycobacterium Celatum from Patients Infected with Human Immunodeficiency Virus,” Clinical Infectious Diseases, Vol. 24, No. 2, 1997, pp. 144-147.

[6] G. Haase, H. Skopnik, S. Bätge and E. C. Böttger, “Cervical Lymphadenitis Caused by Mycobacterium Celatum,” Lancet, Vol. 344, No. 8928, 1994, pp. 1020-1021. http://dx.doi.org/10.1016/S0140-6736(94)91680-2

[7] D. C. Christiansen, G. D. Roberts and R. Patel, "Mycobacterium Celatum, an Emerging Pathogen and Cause of False Positive Amplified Mycobacterium Tuberculosis Direct Test," Diagnostic Microbiology and Infectious Disease, Vol. 49, No. 1, 2004, pp. 19-24.

[8] I. Bux-Gewehr, H. Hagen, S. Ruesch-Gerdes and G. Feurle, "Fatal Pulmonary Infection with Mycobacterium Celatum in an Immunocompetent Patient," Journal of Clinical Microbiology, Vol. 36, No. 2, 1998, pp. 587-588.

[9] J. H. T. Tjhie, A. F. van Belle, M. Dessens-Kroon and D. van Solingen, "Misidentification and Diagnostic Delay Caused by a False-Positive Amplified Mycobacterium Tu- berculosis Direct Test in an Immunocompetent Patient with a Mycobacterium Celatum Infection," Journal of Clinical Microbiology, Vol. 39, No. 6, 2001, pp. 23112312.

http://dx.doi.org/10.1128/JCM.39.6.2311-2312.2001

[10] C. Piersimoni, P. G. Zitti, D. Nista and S. Bornigia, "Mycobacterium Celatum Pulmonary Infection in the Immunocompetent: Case Report and Review,” Emerging Infectious Diseases Journal, Vol. 9, No. 3, 2003, pp. 339-342.

[11] D. Christiansen, G. Roberts and R. Patel, "Mycobacterium Celatum, an Emerging Pathogen and Cause of False Positive Amplified Mycobacterium Tuberculosis Direct Test,”

[12] J. Collazos, E. Martinez and J. Mayo, "Evolution of Serum Beta-2 Microglobulin Concentrations during Treatment of Tuberculosis Patients," Scandinavian Journal of Infectious Diseases, Vol. 31, No. 3, 1999, pp. 265-267. http://dx.doi.org/10.1080/00365549950163554 